\title{
Virtual 3D planning of tracheostomy placement and clinical applicability of 3D cannula design: a three-step study
}

\author{
Bertram J. de Kleijn ${ }^{1,3}$ (1) - Joep Kraeima ${ }^{2}$ (1) Jasper E. Wachters ${ }^{1} \cdot$ Bernard F. A. M. van der Laan ${ }^{1} \cdot$ Jan Wedman $^{1}$. \\ M. J. H. Witjes ${ }^{2}$ (1) Gyorgy B. Halmos ${ }^{1}(1)$
}

Received: 30 August 2017 / Accepted: 16 November 2017 / Published online: 24 November 2017

(c) The Author(s) 2017. This article is an open access publication

\begin{abstract}
Aim We aimed to investigate the potential of 3D virtual planning of tracheostomy tube placement and 3D cannula design to prevent tracheostomy complications due to inadequate cannula position.

Materials and methods 3D models of commercially available cannula were positioned in 3D models of the airway. In study (1), a cohort that underwent tracheostomy between 2013 and 2015 was selected $(n=26)$. The cannula was virtually placed in the airway in the pre-operative CT scan and its position was compared to the cannula position on post-operative CT scans. In study (2), a cohort with neuromuscular disease $(n=14)$ was analyzed. Virtual cannula placing was performed in CT scans and tested if problems could be anticipated. Finally (3), for a patient with Duchenne muscular dystrophy and complications of conventional tracheostomy cannula, a patient-specific cannula was 3D designed, fabricated, and placed.

Results (1) The 3D planned and post-operative tracheostomy position differed significantly. (2) Three groups of patients were identified: (A) normal anatomy; (B) abnormal anatomy, commercially available cannula fits; and (C) abnormal anatomy, custom-made cannula, may be necessary. (3) The position of the custom-designed cannula was optimal and the trachea healed. Conclusions Virtual planning of the tracheostomy did not correlate with actual cannula position. Identifying patients with abnormal airway anatomy in whom commercially available cannula cannot be optimally positioned is advantageous. Patientspecific cannula design based on 3D virtualization of the airway was beneficial in a patient with abnormal airway anatomy.
\end{abstract}

Keywords 3D planning $\cdot$ Cannula $\cdot$ Neuromuscular disease $\cdot$ Tracheostomy

Bertram J. de Kleijn and Joep Kraeima have equally contributed to the manuscript, therefore, sharing first authorship.

Bertram J. de Kleijn

b.j.de.kleijn@umcg.nl

1 Department of Otorhinolaryngology, Head and Neck Surgery, University of Groningen, University Medical Center Groningen, Hanzeplein 1, 9713 GZ Groningen, The Netherlands

2 Department of Oral and Maxillofacial Surgery, Head and Neck Surgery, University of Groningen, University Medical Center Groningen, Hanzeplein 1, 9713 GZ Groningen, The Netherlands

3 Department of Otorhinolaryngology, Head and Neck Surgery, University Medical Center Groningen (UMCG), Hanzeplein 1, PO box 30.001, 9700 RB Groningen, The Netherlands

\section{Introduction}

Tracheostomy is a routine procedure for securing the airway. In most of the cases commercially available, prefabricated tracheostomy cannulas are used to keep the tracheostomy open. These cannulas have fixed variations in size, radius, curvature, and diameter. However, in case of aberrant anatomy of the neck or thorax, the choice of the proper tracheostomy site with the corresponding cannula type can be challenging.

In patients with abnormal anatomy of the neck and thorax, such as, e.g., patients with Duchenne muscular dystrophy, the commercially available cannulas often do not fit due to the extreme scoliosis [1]. Beyond discomfort, suboptimal cannula placement may result in inflammation of the trachea, eventually leading to granulation tissue formation, airway obstruction, or even in fatal complications [2]. Several case reports and our own experience have shown patients who had a fistula of the trachea and 
innominate artery, leading to a fatal hemorrhage [3, 4]. Therefore, proper and cautious cannula selection is essential to reduce these risks.

In general, cannula placement is depending on the estimation of the surgeon during the procedure. Especially, in case of abnormal anatomy, this appraisement is difficult. After surgery, an endoscopic control of the position of the tip of the cannula may be performed to optimize the positioning of the cannula and/or to determine what type of cannula is optimal for the patient. The outcome is subjective and highly depending on the experience of the physician.

Pre-operative visualization of the upper airway with determination of the optimal stoma site could theoretically aid in the surgical planning in case of aberrant neck anatomy. Furthermore, optimal choice of standard cannula or a custom-made cannula based on pre-operative visualization of the upper airway can prevent complications. A three-step approach is used for analysis of the added value of 3D visualization and surgical planning of tracheostomy and cannula placement. As a first step, a series of retrospective cases was analyzed to explore the applicability of pre-operative 3D visualization and predictability of surgical placement. As a second step, different groups of neuromuscular patients were identified using 3D visualization to predict cannula problems. The third step is the implementation of a custom cannula in clinical case including design, fabrication, placement, and evaluation of the cannula position.

\section{Materials and methods}

\section{Virtual cannula placement and comparison with actual post-surgery position}

The first series of patients was selected from a cohort of patients who underwent a tracheostomy at the department of Otolaryngology of the University Medical Center Groningen (UMCG) between 2013 and $2015(N=150)$. Only patients of whom a pre- and post-tracheostomy CT scan of the head and neck was available were included for analysis $(N=26)$. Pre- and post-tracheostomy scans of the patient series were assessed by an Otorhinolaryngologist (GBH) and Technical Physician (JK) using the following inclusion criteria: was the patient positioned in an extended head position? Was the area of tracheostomy entrance (skin) visualized? Was the tip of the sternum visualized? Was the airway visualized? All patient data that did not meet these criteria were excluded from further analysis in this study. Note that no specific scanning protocol was used, as the cases where retrospectively selected. After this quality assessment, ten cases were found to be suitable for inclusion for this study.

The available standard size cannulas, applied in the UMCG, were scanned using a cone beam CT scan, after which a 3D virtual model was made using ProPlan 2.1 (Materialise, Leuven, Belgium) (Fig. 1a). For each patient's CT scan (pre- and post-operative), a 3D virtual representation was made, using ProPlan 2.1, including airway, thorax, and skin visualization. The $3 \mathrm{D}$ cannula models were imported in that $3 \mathrm{D}$ reconstructed model of the patient, allowing virtual cannula placement.

Using the pre-tracheostomy data, the scanned cannulas were carefully virtually placed in the virtual model by the Otorhinolaryngologist and Technical Physician. Optimal positioning was obtained by evaluating, and adjusting, the cannula tip position in relation to the airway. For the posttracheostomy data, the actual location of the cannula was identified on the CT scan.

A systematic 2D virtual landmark comparison provided data regarding the difference between the planned cannula position and the post-operative position. The defined landmarks and measurements are presented in Fig. 1, including the apex of the sternum, tip of the cannula, shoulder point of the cannula, and line between the clavicles. All of which were selected on the mid-sagittal slice through the center of the tip of the cannula and axial slice presenting the body of the cannula. The distance between the sternum and the cannula flange $(D)$ describes the height of the postoperative placement compared to the planned positioning. The distance between the sternum and the cannula tip $(T)$ describes the position of the cannula tip compared to the planned position. The angle of the cannula in the sagittal plane was related to the sternum $(\alpha)$. In the transverse plane, the angle of the cannula was related to a straight line between the clavicles ( $\beta$ ) (Fig. 1).

\section{Identification of tracheostomy cannula-specific airway anatomy in patients with neuromuscular disease}

For the second part of this study, the files of 234 patients treated for neuromuscular disease at the UMCG were reviewed. 14 patients were identified who had CT scan of the head and neck and upper chest, which was applicable for 3D analysis. Most of the patient had no tracheostoma $(n=10)$, some did $(n=3)$, and in one case, pre- and posttracheostomy scans were available. The same $3 \mathrm{D}$ virtual models of scanned cannulas that were used in the first part of the study were used. Optimal cannula positioning was determined using ProPlan CMF 2.1 (Materialise, Leuven, Belgium). First, of every available CT scan, a 3D virtual representation was made. Comparable to the first group, a 


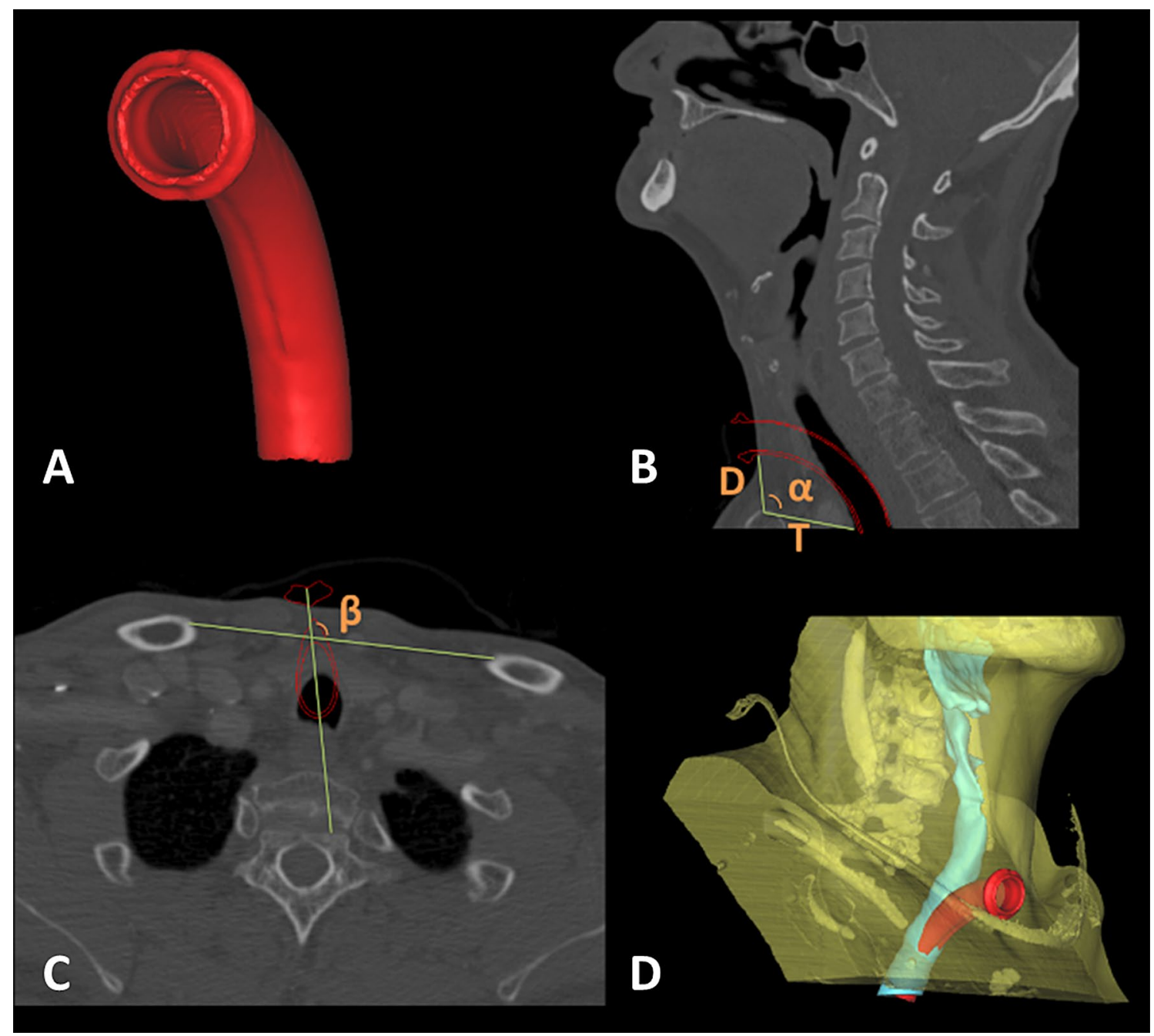

Fig. 1 Analysis in 2D images of scans using described distances. $D$ the distance between the sternum and the cannula flange. $T$ the distance between the sternum and the cannula tip. $\alpha$ the angle of the cannula in the sagittal plane related to the sternum. $(\beta)$ the angle of the cannula related to a straight line between the clavicles in the trans-

3D cannula model was imported in that 3D reconstruction and optimal placement was virtually determined. Cannula was positioned as optimally as possible, and the tip of the cannula was tried to be centered in the tracheal lumen and the shield on skin of the lower neck.

\section{Evaluation of a 3D designed, fabricated, and placed cannula: report of a case}

A 42-year-old male patient suffering from Duchenne muscular dystrophy was identified with granulation tissue formation of the trachea due to inadequate cannula position. The patient was not followed up by an otolaryngologist for multiple years and this situation was very likely due to the progression of kyphoscoliosis. Several different brands, verse plane. a 3D-rendered cannula based on CT data. b Sagittal view with variables $D, T$, and $\alpha$. c Transverse view with variable $\beta$. d 3D-rendered view bases on CT data with 3D-rendered cannula in optimal position

types, and sizes of cannulas were tried, but none had an optimal position. Attempts to optimize positioning using extra spacers between the cannula and the skin failed, too. A CT scan of the airway (with the cannula in place) was made. For the custom cannula design, a 3D airway model was made based on the CT scan using Proplan CMF 2.1. The conventional cannula that the patients were using was segmented to a 3D model, as well as the bony structures and the skin of the patient for anatomical reference. The optimal entrance point in the trachea and the caudal tip location of the custom cannula was agreed on by the surgeon (GBH) and technical physician (JK). The radius and diameter of the cannula were determined on the 3D models and the cannula was designed using 3-Matic 10.0 (Materialise, Leuven, Belgium). The final design was sent both as stl-files and a 
3D-printed physical model to a producer of custom-made silver cannulas. The cannula was produced from silver which conforms the dimensions of the 3D design. The cannula was placed and its position was checked by a CT scan and by fiberoptic endoscopy.

\section{Statistical analysis}

For the first part of this study, distances were explored using the Kolmogorov-Smirnov test. They were normally distributed and presented as mean \pm standard deviation. A paired $T$ test was used to determine statistical differences. For the second part and third part of the study, no statistical analysis was possible.

\section{Results}

\section{Virtual cannula placement and comparison with actual position}

The patients' characteristics of the first part of the study are described in Table 1. The average age of the patients was 58 years (range 38-75 years). There were 7 males and 3 females.

The results of the analysis performed are presented in Table 2. Post-operative cannula placement was different from the pre-operative 'optimal' placement. The largest deviation was found between the tip of the sternum and the flange of the cannula $(D)$ on the virtually and operatively placed cannulas, with an average of $14.23 \mathrm{~mm}$. The difference of the tip of the cannula to the tip of the sternum $(T)$ was found to be smaller, with an average of $5.6 \mathrm{~mm}$. Both $D$ and $T$ values between the pre- and post-operative situations were statistically significantly different $(p<0.001$ and $p=0.002$, respectively). The measured angles show that the angulation of the cannula in transversal view is mostly not different from pre-operative planning (average difference $\left.1.51^{\circ}, \mathrm{ns}\right)$, but the angulation in axial view does significantly differ (average difference $17.36^{\circ}, p=0.019$ ).

\section{Identification of tracheostomy cannula-specific airway anatomy in patients with neuromuscular disease}

The patients' characteristics of the second part of the study are described in Table 3 . The average age of the patients was 24 years (range 17-42 years). There were 13 males and 1 female.

Three-dimensional placement of the conventional cannulas and evaluation of their position identified three groups of patients: (1) normal or close to normal anatomy and a commercially available cannula would fit $(n=7)$. (2) The anatomy is abnormal, but a standard cannula would fit $(n=3)$. (3) The anatomy is abnormal, and a standard cannula would not fit $(n=4)$.
Table 1 Patient characteristics of patients in study 1 with normal anatomy
Table 2 Variables showing the measured distances and angles of the cannula position in patients with normal anatomy

\begin{tabular}{lllllll}
\hline & $\begin{array}{l}\text { Age at } \\
\text { tracheos- } \\
\text { tomy }\end{array}$ & Sex & Reason for tracheostomy & $\begin{array}{l}\text { Duration of } \\
\text { cannula use }\end{array}$ & $\begin{array}{l}\text { Complications due } \\
\text { to tracheostomy? }\end{array}$ & Type of cannula \\
\hline 1 & 61 & M & Oncology & 7 months & No & Tracoe 10 \\
2 & 43 & M & Trauma & 12 days & No & Shiley 8 \\
3 & 73 & M & Laryngeal paralysis & 2 months & No & Shiley 8 \\
4 & 38 & M & Lymphoma & 2.5 months & No & Tracoe 8 \\
5 & 75 & M & Oncology & 2 months & No & Tracoe 9 \\
6 & 58 & F & Oncology & 8 months & No & Tracoe 6 \\
7 & 68 & M & Post radiation fibrosis & 10 months & No & Tracoe 10 \\
8 & 61 & F & Oncology & 1 months & No & Tracoe 8 \\
9 & 53 & F & Oncology & 1 months & No & Shiley 8 \\
10 & 50 & M & Oncology & 4 months & No & Tracoe 8 \\
\hline
\end{tabular}

\begin{tabular}{lllll}
\hline & $\mathrm{D}(\mathrm{mm})$ & $\mathrm{T}(\mathrm{mm})$ & $\mathrm{A}\left({ }^{\mathrm{o}}\right.$ degrees $)$ & $\mathrm{B}\left({ }^{\mathrm{o}}\right.$ degrees $)$ \\
\hline Pre-operative & $23.5 \pm 5.51$ & $41.06 \pm 5.04$ & $140.77 \pm 28.84$ & $86.54 \pm 7.33$ \\
Post-operative & $37.75 \pm 4.02$ & $35.46 \pm 5.16$ & $123.42 \pm 28.56$ & $88.04 \pm 23.5$ \\
Mean difference & $14.23 \pm 5.48$ & $5.60 \pm 4.11$ & $17.36 \pm 19.22$ & $1.51 \pm 22.49$ \\
$T$ test & $p<0.001$ & $p=0.002$ & $p=0.019$ & $p=0.837$ \\
\hline
\end{tabular}

For details, see "Materials and methods" (mean \pm standard deviation) 
Table 3 Patient characteristics of patients of study 2 with neuromuscular disease

\begin{tabular}{|c|c|c|c|c|c|c|}
\hline & Neuromuscular disease & $\begin{array}{l}\text { Age (at time of } \\
\text { scan) }\end{array}$ & Sex & Tracheostomy? & $\begin{array}{l}\text { Complications due to } \\
\text { tracheostomy? }\end{array}$ & Type of cannula \\
\hline 1 & Spinal muscular atrophy & 33 & M & No & N.A. & N.A. \\
\hline 2 & Spinal muscular atrophy & 25 & $\mathrm{~F}$ & No & N.A. & N.A. \\
\hline 3 & M. Duchenne & 19 & M & No & N.A. & N.A. \\
\hline 4 & M. Becker & 22 & M & Yes & Yes, granulation & Rusch \\
\hline 5 & M. Becker & 40 & M & No & N.A. & N.A. \\
\hline 6 & Spinal muscular atrophy & 24 & M & No & N.A. & N.A. \\
\hline 7 & M. Duchenne & 16 & M & No & N.A. & N.A. \\
\hline 8 & M. Duchenne & 28 & M & Yes & Yes, ulcer & Custom-made Bivona 7 \\
\hline 9 & M. Duchenne & 21 & M & Yes & No & Custom-made Bivona \\
\hline 10 & M. Duchenne & 17 & M & No & N.A. & N.A. \\
\hline 11 & M. Steinert & 19 & M & No & N.A. & N.A. \\
\hline 12 & M. Duchenne & 42 & M & Yes & Yes, granulation & Tracoe 8 \\
\hline 13 & M. Duchenne & 19 & M & No & N.A. & N.A. \\
\hline 14 & M. Duchenne & 14 & M & No & N.A. & N.A. \\
\hline
\end{tabular}
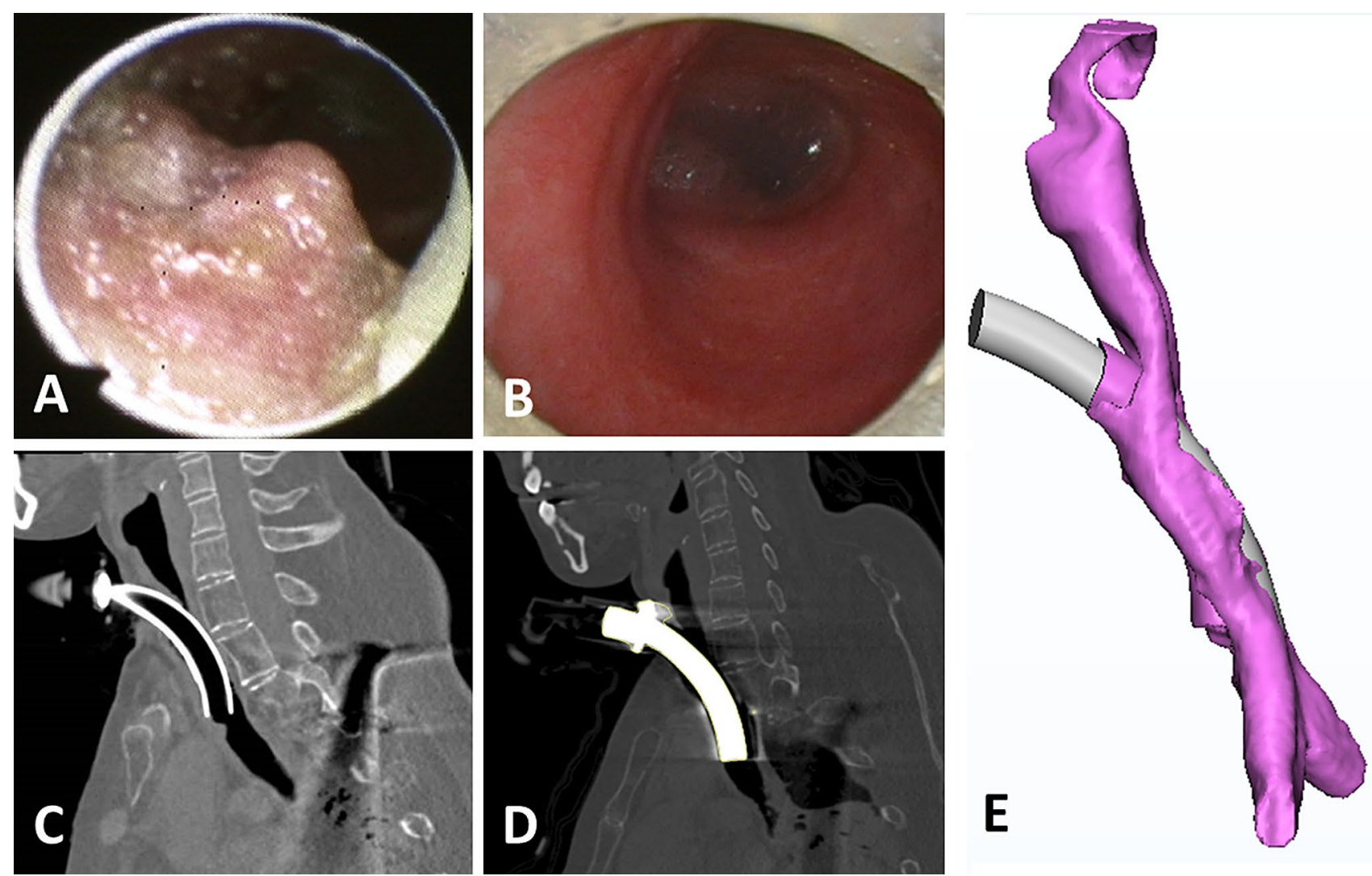

nula. c Sagittal CT view of the position of the standard cannula. d

Fig. 2 Endoscopic (a, b), CT (c, d), and 3D images (e) of the patient with conventional and with 3D-designed cannula. a, c With conventional cannula, b, d with 3D-designed cannula. a Endoscopic view through the standard cannula with granulation formation in the trachea. b Endoscopic view of the trachea through the 3D-designed can-
Sagittal CT view of the position of the 3D-designed cannula. e Segmented airway with the conventional cannula in situ and with the 3D-designed cannula 


\section{Evaluation of a 3D designed, fabricated, and placed cannula: report of a case}

This patient is the first to receive a custom-made cannula based on 3D planning. Comparing the CT scan with the old cannula to the CT scan with the new cannula, the improvement in positioning can be seen (Fig. 2). The complaints of the patients were immediately dissolved. Two months after placement of the cannula, the granulation tissue healed and the cannula positioning was centered in the airway without any signs of decubitus off the trachea (Fig. 2).

\section{Discussion}

In this three-step study, using 3D technology, (1) we have a setup created for the 3D analysis of tracheostomy cannula and their placement, (2) we have identified cannulaspecific airway anatomy in patients with neuromuscular diseases, and (3) successfully designed, produced, and placed a 3D-planned cannula in a patients after several previous failures of custom-made cannula.

In our study, we show that the virtual planning differs statistically significantly from the actual cannula placement assessed on the post-operative CT scan. Several factors attributed to this difference. First, because of the retrospective nature of the study, the acting surgeon was not informed on a possible ideal position of the stoma and choice of cannula. Second, superimposing the pre- and post-op scans was difficult due to different neck extension and subsequent different scanning position. Third, the proper surgical route is not easy to predict due to soft tissues of the neck and, therefore, flexibility the tissues around the stoma.

In our series of patients with neuromuscular disease, we could identify three sorts of anatomical situations regarding virtual cannula placement: (1) the anatomy is not abnormal and a standard cannula would fit. (2) The anatomy is abnormal, but a standard cannula would fit. (3) The anatomy is abnormal and a standard cannula would not fit. The assessment of cannula fit in this group was made by trying to place custom-made cannulas in a 3D model of the airway. This assessment did not suffer from the problem found in the first study in which cannula placement was tried to be predicted from a 3D model. In the second study, the cannula was fit in any position possible. These important findings could help pre-operatively in identifying patients who might get complications after tracheostomy. These patients could benefit from individualized, 3D-designed cannulas.

In this study, we show that it is possible to design a custom-made cannula using 3D virtualization techniques for patients with abnormal anatomy and/or suboptimal cannula positioning.
Especially, in patient with neuromuscular diseases such as Duchenne muscular dystrophy, suboptimal cannula placement can lead to mild or severe complications [1-4]. As the disease progresses, the progressive changes in anatomy can worsen problems with cannula positioning. The custom design of cannulas can provide a definitive solution for patients in whom optimal care cannot be achieved by the commercially available cannulas.

No studies have been reported on tracheostomy planning and cannula placement using virtual 3D planning. It is apparent that the use of 3D visualization and subsequent development of surgical techniques and 3D printed aids and parts is the next logical step in the treatment of patients with complex tracheal anatomy.

Virtual 3D reconstructions have been used before in otorhinolaryngology, using stereophotogrammetrical analyses or CT scans [5-8]. Applications vary from laryngectomy stoma assessment to 3D planning of operations. The overview article of Kaye et al. summarizes the use of 3D printing for educational purposes, auricular prosthesis, 3D-printed hearing aids, surgical planning, and managing the pediatric airway with 3D models of the airway to optimize stent placement [9].

When determining the optimal cannula position from pre-operative scans, sometimes, a compromise had to be made between the positioning of the cannula tip in the trachea and the position of the flange of the cannula on the skin. The tip was used for alignment, sometimes meaning that the cannula flange did not align with the skin. In some cases, this led to unrealistic cannula placement with standard sized cannulas. We think that for these cases, patientspecific 3D-designed cannulas could aid in obtaining the optimal cannula position.

The analysis of the series of patients with neuromuscular diseases did not allow statistical analysis. However, the findings are very relevant, because they could help identifying patients who might get complications after tracheostomy. Individualized, 3D-designed cannulas could help these patients.

This is the first report of using 3D virtualization techniques to design a custom-made silver cannula for a patient with abnormal anatomy and cannula problems. The outcome in this patient shows that perfect positioning could be achieved by this technique and complications of suboptimal positioning can be prevented.

To determine if pre-operative planning can influence the surgical outcome, a prospective study is necessary. If superimposing of pre- and post-operative CT scans is more precise using a predefined scan protocol, further analysis for 3D planning of the tracheostomy site and cannulas can be done. 


\section{Conclusions}

In this study, the use of virtual tracheostomy cannula placement using 3D reconstructions of CT scans was explored. We found that the pre-operative positioning the stoma and subsequent placement of a cannula on a 3D model from the airway did not match with the surgical outcome. However, when stoma placement was not considered, we could identify a group of patients with neuromuscular disease, and abnormal anatomy of the neck that might benefit from 3D design of the cannula, using a post-tracheostomy CT scan. This was supported by our case that showed that customdesigned cannula using $3 \mathrm{D}$ virtualization techniques can reduce complications of suboptimal cannula positioning.

For optimal 3D virtual planning of the tracheostomy site and cannula position, a prospective study is required. This study provides the first data towards individual tracheostomy and cannula placement planning in patients with abnormal anatomy of the head and neck.

\section{Compliance with ethical standards}

Funding This study was not funded.

Conflict of interest There are no conflicts of interest.

Ethical approval The study has been reviewed by the Institutional Review Board of the UMCG and a consent waiver was granted for this retrospective chart review. Therefore, no informed consent was needed according to Dutch law.

Informed consent Informed consent was obtained from all individual participants for whom identifying information is included in this article.

Open Access This article is distributed under the terms of the Creative Commons Attribution 4.0 International License (http://creativecommons.org/licenses/by/4.0/), which permits unrestricted use, distribution, and reproduction in any medium, provided you give appropriate credit to the original author(s) and the source, provide a link to the Creative Commons license, and indicate if changes were made.

\section{References}

1. Meinesz AF, Bladder G, Goorhuis JF, Fock JM, Staal-Schreinemachers AL, Zijlstra JG, Wijkstra PJ (2007) 18 years experience with mechanical ventilation in patients with Duchenne muscular dystrophy. Ned Tijdschr Geneeskd 151:1830-1833

2. Ilan O, Gross M, Zaltzman Y, Sasson A, Marcus EL (2015) Diagnosis and conservative management of late tracheotomy complications in chronic ventilator-dependent patients. Head Neck 37:716-721

3. Bekele A, Tesfaye S (2015) Fatal Tracheo-Innominate artery fistula associated with a tracheostomy tube at a teaching university hospital in addis ababa: case report and review of literature. Ethiop Med J 53:155-158

4. Saito T, Sawabata N, Matsumura T, Nozaki S, Fujimura H, Shinno S (2006) Tracheo-arterial fistula in tracheostomy patients with Duchenne muscular dystrophy. Brain Dev 28:223-227

5. Dirven R, Wouters Y, Vreeken R, Maal TJ, Marres HA (2012) Three-dimensional stereophotogrammetrical analysis of peristomal fixation of adhesive base plates during hands-free speech among laryngectomised patients related to tracheostoma volumes. Clin Otolaryngol 37:124-129

6. Fujimoto T, Imai K, Matsumoto H, Sakamoto H, Nakano T (2011) Tracheobronchial anomalies in syndromic craniosynostosis with 3-dimensional CT image and bronchoscopy. J Craniofac Surg 22:1579-1583

7. Schepers RH, Raghoebar GM, Vissink A et al (2013) Fully 3-dimensional digitally planned reconstruction of a mandible with a free vascularized fibula and immediate placement of an implantsupported prosthetic construction. Head Neck 35:E109-E114

8. Barrera JE (2014) Virtual surgical planning improves surgical outcome measures in obstructive sleep apnea surgery. Laryngoscope 124:1259-1266

9. Kaye R, Goldstein T, Zeltsman D, Grande DA, Smith LP (2016) Three dimensional printing: a review on the utility within medicine and otolaryngology. Int J Pediatr Otorhinolaryngol 89:145-148 